\title{
Consumo de alcohol, síntomas neurológicos y retos diagnósticos en un paciente con una gastrostomía percutánea
}

\author{
Alcohol consumption, neurological symptoms \\ and diagnostic challenges in a patient with a \\ percutaneous endoscopic gastrostomy
}

\author{
Pablo Barrio*, Marzia Merlino**, Mercè Balcells-Oliveró*. \\ * Instituto Clínic de Neurociencias, Servicio de Psiquiatría, Unidad de Conductas Adictivas. Hospital Clínic, Barcelona. España. \\ ** Unidad de Psiquiatría, Facultad de Ciencias Biomédicas y Dentales e Imagen Morfofuncional. Universidad de Messina, \\ Messina. Italia.
}

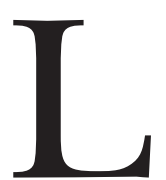
a adicción es un fenómeno de gran complejidad que conlleva manifestaciones en un amplio rango de dimensiones, incluyendo psicológicas, conductuales, médicas y socioeconómicas. Muchas teorías han intentado explicar las conductas extremas que la adicción impone en los individuos afectados. No obstante, aún estamos lejos de llegar a una explicación definitiva y global capaz de unificar todos los aspectos subyacentes de la adicción (Nutt, Lingford-Hughes, Erritzoe y Stokes, 2015; Pober, 2013).

En la lista de sustancias más consumidas a nivel mundial, el alcohol aparece segundo, detrás de la nicotina. Presentamos aquí un estudio de caso que describe un fenómeno conductual complejo en un paciente con dependencia de alcohol. Ilustra algunas de las características claves de la adicción.

El paciente es un hombre de 60 años que llevaba una gastrostomía endoscópica percutánea para alimentación debido a cáncer orofaríngeo que requirió una bucofaringectomía y una hemiglosectomía, además de una traqueotomía en 1999. El paciente también sufre de un trastorno por uso de alcohol de larga duración.

$\mathrm{Al}$ principio del 2005, el paciente fue admitido a Urgencias por un episodio agudo con ataxia, una disminución del estado de alerta, disartria y nistagmo en ambos ojos en todas las direcciones de la mirada. Varias pruebas complementarias (TAC, analítica sanguínea, ECG, EEG) no hallaron anomalías. Los síntomas remitieron en menos de 24 horas. Durante el 2005, el paciente fue hospitalizado otra vez con los mismos síntomas. Igual que anteriormente, este episodio fue autolimitado y las pruebas complementarias no detectaron anormalidades relevantes.

En el 2006, el paciente fue ingresado en el servicio de Neurología para estudiar lo que se consideraron episodios recurrentes de encefalopatía. Una vez más, varias pruebas complementarias (SNC, IRM, EEG, ecodópler de tronco supraaórtico, punción lumbar) no obtuvieron resultados importantes.

Se atribuyó al cuadro clínico un posible origen epiléptico, por lo que se le trató con levetiracetam $1000 \mathrm{mg} /$ día. Posteriormente se cambió el tratamiento a ácido valproico y, por último, en el 2008, a carbamazepina $600 \mathrm{mg}$ /día. A pesar de ello, fue admitido varias veces en Urgencias por los mismos síntomas neurológicos entre 2006 y 2009.

El diagnóstico definitivo se realizó en agosto 2009. El paciente se había estado autoinyectando grandes cantidades de bebidas alcohólicas a través de su gastrostomía endoscópica percutánea, un hecho desconocido hasta ese momento. El paciente comenzó tratamiento ambulatorio, tanto psicológico como farmacológico, en la Unidad de Conductas Adictivas. No obstante, logró la abstinencia solo durante periodos breves. También presentó algunas complicaciones, como una hemorragia gástrica tras beber alcohol mientras estaba medicado con disulfiram.

Es importante tener en cuenta algunas consideraciones al revisar este caso. Primero, la vía de administración inusual. Además de ser una manera muy infrecuente de ingerir bebidas alcohólicas, la gastrostomía endoscópica per-

Recibido: Mayo 2019; Aceptado: Noviembre 2019.

Enviar correspondencia a:

Pablo Barrio. Unidad de Conductas Adictivas, Hospital Clínico de Barcelona. Calle Villarroel 17008036 Barcelona, España.

E-mail: pbarrio@clinic.cat. 
cutánea permite alcanzar niveles altos de concentración de alcohol en sangre. Este hecho originó los síntomas neurológicos manifestados por el paciente en varias ocasiones. Aunque se han descrito otras vías de ingestión de alcohol, son altamente infrecuentes (Stogner, Eassey, Baldwin y Miller, 2014).

Segundo, a pesar de que el historial médico del paciente incluía condiciones claramente asociadas con la ingestión actual de alcohol (dependencia del alcohol y cáncer bucofaríngeo), pareció razonable omitir la consideración del alcohol durante el proceso de diagnóstico diferencial porque su estado físico le impedía ingerir bebida alguna. Además, había ausencia de algunos de los síntomas clásicos, como el fetor, que permiten reconocer la intoxicación por alcohol. En conjunto, a pesar de la sencillez de la solución final de este puzle, el proceso diagnóstico claramente supuso un reto. Prueba de esto son los 4 años de seguimiento por parte del servicio de Neurología, los diversos tratamientos farmacológicos aplicados y la cantidad de pruebas complementarias realizadas.

Por último, este es un caso adecuado para ilustrar la naturaleza de la adicción en sí, donde la ingestión de la sustancia se convierte en primordial, indistintamente de las dificultades y sus consecuencias.

En conclusión, pensamos que este caso revela cómo el alcohol, una sustancia consumida con tanta frecuencia en nuestra sociedad, debe ser un elemento importante al realizar varios diagnósticos diferenciales, en particular aquellos que implican síntomas neurológicos, como estupor, ataxia y nistagmo.

\section{Conflicto de interés}

Los autores declaran la inexistencia de conflicto de interés.

\section{Referencias}

Nutt, D. J., Lingford-Hughes, A., Erritzoe, D. y Stokes, P. (2015). The dopamine theory of addiction: 40 years of highs and lows. Nature Reviews Neuroscience, 16, 305-312. doi:10.1038/nrn3939.

Pober, J. M. (2013). Addiction is not a natural kind. Frontiers in Psychiatry, 4, 123. doi:10.3389/fpsyt.2013.00123.

Stogner, J. M., Eassey, J. M., Baldwin, J. M. y Miller, B. L. (2014). Innovative alcohol use: Assessing the prevalence of alcohol without liquid and other non-oral routes of alcohol administration. Drug and Alcohol Dependence, 142, 74-78. doi:10.1016/j.drugalcdep.2014.05.026. 